\title{
Chronic Pulmonary Aspergillosis: An Update on Diagnosis and Treatment
}

\author{
Cendrine Godet $^{\mathrm{a}}$ Bruno Philippe ${ }^{\mathrm{b}} \quad$ François Laurent $^{\mathrm{c}}$ Jacques Cadranel ${ }^{\mathrm{d}}$ \\ ${ }^{a}$ Department of Infectious Diseases, CHU la Milétrie, Poitiers, ${ }^{b}$ Department of Pneumology, René Dubos \\ Hospital, Pontoise, ' Department of Diagnostic and Therapeutic Imaging, Haut-Levêque Hospital, University \\ Hospital of Bordeaux and Université Victor Segalen, Bordeaux, and ${ }^{\mathrm{d}}$ Service de Pneumologie, Hôpital Tenon \\ AP-HP and Faculté de Médecine Pierre et Marie Curie, Université Paris 6, Paris, France
}

\section{Key Words}

Chronic pulmonary aspergillosis $\cdot$ Aspergilloma $\cdot$ Antifungal treatment

\section{Abstract}

Chronic pulmonary aspergillosis (CPA) affects individuals with non-systemic or mildly systemic immunodepression or altered pulmonary integrity due to underlying disease. It has been reported with a variety of clinical and radiological patterns. The condition should be distinguished from simple aspergilloma and allergic bronchopulmonary aspergillosis as well as invasive aspergillosis in severely immunocompromised patients. CPA generally requires long-term antifungal treatment and surgery may be considered. Life-threatening haemoptysis may be prevented by bronchial arteriography with embolisation. However, currently there are no documented treatment recommendations for CPA. This review provides an up-to-date practical overview of this condition, including a comprehensive update on diagnosis and management.

(c) 2014 S. Karger AG, Basel

\section{Introduction}

Bronchopulmonary aspergillosis is caused by the inhalation of spores from Aspergillus spp., most commonly Aspergillus fumigatus [1], although similar clinical and radiological presentations can also be attributed to other fungi. The clinical manifestations of the condition are largely determined by the integrity of the lung [2] as well as the innate and adaptive immune responses of the individual to the inhalation of the fungal spores [3]. The condition is broadly divided into different clinical forms depending on the immunological status of the patient and lung: allergic bronchopulmonary aspergillosis (ABPA) is caused by the bronchial colonisation of Aspergillus spp. and a T-helper cell 2 immune response due to lung hypersensitivity in patients with asthma and cystic fibrosis, whereas acute invasive pulmonary aspergillosis (IPA) affects severely immunocompromised patients [4]. In contrast, the chronic forms of Aspergillus infection constitute a debilitating syndrome affecting patients with pre-existing structural lung diseases (fig. 1) $[3,5]$.

Chronic pulmonary aspergillosis (CPA) occurs in individuals with an underlying lung disease, in particular,

\section{KARGER}

E-Mail karger@karger.com www.karger.com/res
(C) 2014 S. Karger AG, Basel

0025-7931/14/0882-0162\$39.50/0
Prof. Jacques Cadranel

Service de Pneumologie, Hôpital Tenon 4 Rue de la Chine

FR-75970 Paris, Cedex 20 (France)

E-Mail jacques.cadranel@tnn.aphp.fr 
Table 1. CPA: diagnosis criteria and definitions [3]

1 Chronic pulmonary or general symptoms including at least 1 of the following (for a minimum of 3 months in duration): weight loss, productive cough or haemoptysis

2 A progressive formation and expansion of single or multiple pulmonary cavitations surrounded by a wall and possible pleural thickening on radio-imaging $[20,27]$

3 A positive result for a serum Aspergillus spp. precipitins test or an isolation of Aspergillus spp. from the pulmonary or pleural cavity

4 Increased biological inflammatory syndrome markers (C-reactive protein, plasma viscosity or erythrocyte sedimentation rate)

5 The exclusion of all other causes that could imitate the symptoms (bronchial carcinoma, TB and atypical mycobacteria)

6 No overt immunocompromising conditions (HIV infection, leukaemia and chronic granulomatous disease)

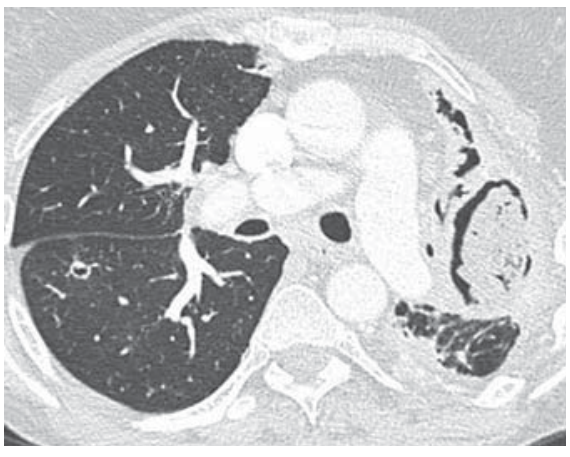

Fig. 1. CPA in a 50-year-old patient. A large cavity filled with a typical fungus ball surrounded by an air crescent is seen in a partly collapsed left upper lobe. The pleura are thickened and the remaining left lung shows distortion features.

chronic obstructive pulmonary disease (COPD) [6]. Unlike IPA, CPA affects individuals who are not (or are mildly) immunocompromised or are locally pulmonaryimmunocompromised due to underlying disease. The condition is associated with high morbidity and mortality. It usually requires long-term antifungal treatment, which transforms disease prognosis in many cases $[7,8]$. This review will systematically explore the current classifications, diagnostic procedures, risk factors and treatment strategies for CPA.

Chronic Pulmonary Aspergillosis
Table 2. Methods for diagnosing CPA

Clinical examination for risk factors

Alcoholism, tobacco abuse, diabetes, corticosteroid use,

COPD or undernourishment

ICU patients, patients with cirrhosis

Chest X-ray and CT

Important for a presumptive diagnosis

Radiological appearance described as simple or complex aspergilloma

Serological testing

Sputum, bronchoscopy or bronchoscopy with BAL

Direct examination and culture

Detection of GM in $\mathrm{BAL}^{1}$

Biopsy sample (perfibroscopic or percutaneous TTNA biopsy)

With histological analysis or microbiological culture

Video-assisted thoracoscopy

Detection of GM in serum ${ }^{2}$

TTNA $=$ Transthoracic needle aspiration.

${ }^{1}$ Confirmatory studies are needed [9].

${ }^{2}$ In forms of CNPA with a semi-invasive nature, the antigen can sometimes be positive for GM $[10,11]$.

\section{Methods}

This review involved a search of the literature on CPA. We searched PubMed using the terms 'Aspergillus, chronic pulmonary aspergillosis, chronic cavitary pulmonary aspergillosis, chronic necrotising pulmonary aspergillosis, chronic fibrosing pulmonary aspergillosis (CFPA), aspergilloma, Infectious Diseases Society of America (IDSA) guidelines, surgical treatment, antifungal therapy, haemoptysis, management and diagnosis', for articles published in English up to August 2013. We hand-searched reference lists from clinically relevant articles for other articles. Titles were screened and abstracts were read to select relevant articles.

\section{Diagnosis Approach}

The diagnosis of CPA relies on a combination of criteria related to patient characteristics, thoracic CT scan findings and mycological analysis, i.e. the detection of Aspergillus precipitins (IgG) in the serum and/or the isolation of Aspergillus spp. from respiratory samples [3] (table 1). However, none of these criteria in isolation is specific for CPA. Each can be observed individually in cases of lung cancer or other chronic respiratory infections such as tuberculosis (TB) or non-TB mycobacterial infection but also in a typically complete form of CPA (table 1). Consequently, the isolation of Aspergillus spp. may often only be the evidence of colonisation of the respiratory tract, especially as the sensitivity/specificity of galac- 
Table 3. Frequency of underlying condition in CPA [6]

\begin{tabular}{lcc}
\hline \multirow{2}{*}{ Underlying condition } & \multicolumn{2}{c}{ Frequency in 126 CPA cases } \\
\cline { 2 - 3 } & total (\%) & primary (\%) \\
\hline Classic TB & $21(16.7)$ & $20(15.9)$ \\
Non-TB mycobacterial infection & $20(15.9)$ & $18(14.3)$ \\
ABPA \pm asthma & $18(14.3)$ & $15(11.9)$ \\
COPD and/or emphysema \pm bullae & $42(33.3)$ & $12(9.5)$ \\
Pneumothorax \pm bullae & $21(16.7)$ & $12(9.5)$ \\
Lung cancer survivor & $13(10.3)$ & $12(9.5)$ \\
Pneumonia ${ }^{1}$ & $28(22.2)$ & $10(7.9)$ \\
Sarcoidosis & $9(7.1)$ & $9(7.1)$ \\
Thoracic surgery & $18(14.3)$ & $6(4.8)$ \\
Rheumatoid arthritis $\quad$ & \\
$\quad$ (no immunosuppression) & $5(4.0)$ & $4(3.2)$ \\
Asthma, no ABPA or SAFS & $13(10.3)$ & $3(2.4)$ \\
SAFS + asthma & $3(2.4)$ & $2(1.6)$ \\
Bullae, no COPD and no pneumothorax & $2(1.6)$ & $1(0.8)$ \\
Sub-acute invasive aspergillosis & $1(0.8)$ & $1(0.8)$ \\
Ankylosing spondylitis/kyphoscoliosis & $5(4.0)$ & 0 \\
Other & $7(5.6)$ & 0 \\
None & $1(0.8)$ & $1(0.8)$ \\
Total (n) & 232 & 126 \\
\hline
\end{tabular}

SAFS $=$ Severe asthma with fungal sensitisation.

${ }^{1}$ Community-acquired pneumonia requiring hospitalisation.

tomannan (GM) antigen testing (in the serum and respiratory secretions) for the diagnosis of CPA has still to be elucidated. There are a number of diagnostic approaches which can be implemented to identify suspected CPA (table 2).

\section{Clinical Examination and Underlying Risk Factors for CPA}

CPA tends to affect middle-aged patients who are not immunosuppressed. It has an indolent progressive course which can last for years. The usual clinical signs and constitutional symptoms include fever, malaise, fatigue and weight loss. In addition, patients may exhibit non-specific respiratory symptoms such as chronic productive cough and haemoptysis. Pleural thickening and Aspergillus empyema can complicate the course of some forms of CPA.

In almost all cases, it develops as a result of an underlying pulmonary pathology, which may be responsible for the presence of a residual bronchopulmonary or, less frequently, pleural cavity [12]. The two most common conditions associated with the development of CPA are (1) $\mathrm{TB}$ and atypical Mycobacterium infection and (2) COPD and emphysema. The latter are more frequent than the former in recent studies that reflect the decrease of TB epidemics [6]. Other conditions include lung cancer, stage III or IV fibrocystic pulmonary sarcoidosis and silicosis (table 3) [6, 12-14].

The pre-existent cavity is sometimes the only condition underlying the development of a CPA. In other circumstances, a number of comorbidities (that define a socalled 'mild immunosuppression') represented by alcoholism, tobacco abuse, diabetes, glucocorticoid treatment or TNF- $\alpha$ inhibitor therapy are found [3]. In any case, the CPA forms are characterised by the absence of vascular invasion. At most, a moderate wall invasion of the cavity by hyphae (as opposed to the invasive forms of immunocompromised subjects), a low tendency for metastasis and a chronic granulomatous reaction may occur [12].

\section{The Role of CT Scan Imaging in CPA Diagnosis}

Radiological features are a combination of the underlying structural lung parenchyma alterations resulting mostly from the lung immune/inflammatory response against Aspergillus spp. rather than from direct invasion by the fungi. Yousem [15] reported three pathological patterns: a necrotising granulomatous pneumonia, a granulomatous bronchiectatic cavity with parenchymal invasion and a bronchocentric granulomatosis-like reaction. These histopathological descriptions indicate the variety of radiological patterns observed.

Initial descriptions of CPA have been performed using chest X-rays [16-18]. CT has provided useful information regarding the characteristics, distribution and extent of features in great detail and with precision $[19,20]$. These findings are non-specific and can be encountered in TB, actinomyosis and lung carcinoma. Nevertheless, in a proper clinical setting, the diagnosis of CPA can be suggested. The most common appearance is the presence of unilateral or bilateral areas of consolidation, frequently with one or several cavitations. In about $50 \%$ of cases, these contain typical fungus balls [5] that appear as lowattenuation material that does not enhance after intravenous (IV) injection of contrast media (fig. 1). The walls of cavitation are thick and the pleura are frequently also thickened; these features are useful for distinguishing CPA from simple aspergilloma which develops in a previous, known thin-walled cavity. Additional features of varying degrees are areas of consolidation or of groundglass attenuation, most often seen in the cavity surroundings. Pulmonary nodules of varying sizes may be present, sometimes taking the characteristic appearance of the tree-in-bud sign, indicating that small dilated bronchi- 


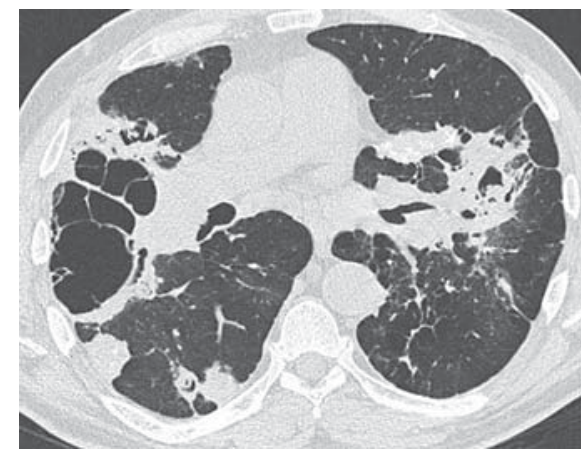

Fig. 2. CPA in a patient on long-term corticosteroid treatment for COPD. Multiple cavities are seen in both lungs within areas of consolidation. Small air-crescent findings are visible in nodular areas and features of advanced emphysema are present.

oles are filled by exudate and that inflammatory changes are developing (fig. 1). These features are frequently unilateral or asymmetric and are prominent in the lung or the part of the lung with cavities. The upper lobe is most often involved and frequently appears collapsed, sometimes severely so. Additional features are related to the underlying pulmonary disease, e.g. $\mathrm{TB}$, sarcoidosis or COPD. However, features of pulmonary fibrosis, distortion, traction bronchiectasis and parenchymal scarring may also be related to the development of a particular form of the disease, so-called CFPA [3] (fig. 2). Several patterns of imaging features and evolution have been described in a clinico-radiological classification by Denning et al. [3].

\section{Mycological and Immunological Diagnosis of CPA}

While sputum samples can reveal the presence of $A s$ pergillus spp., a negative result can be found in 50\% of cases [4]. The detection of filamentous fungi evoking Aspergillus by direct examination in bronchial samples is significantly associated with CPA compared to colonisation [21]. Bronchoalveolar lavage (BAL) sensitivity is usually about $50 \%$ in most cases, although it can be difficult to distinguish between colonisation and infection $[21,22]$. Colonisation is less probable in cases with radiological findings consistent with CPA. A study by Ohba et al. [23] found $67.4 \%$ of the patients to have Aspergillus spp. colonisation, but only $32.6 \%$ were diagnosed with CPA. This study highlights the importance of identifying additional clinical and radiological characteristics, such as the underlying disease as well as positivity for Aspergillus spp. antibodies, for obtaining a definite diagnosis of CPA.

Chronic Pulmonary Aspergillosis
The impact of GM antigen testing (in the serum and respiratory secretions) for the diagnosis of CPA has still to be elucidated. A comparison of the usefulness of testing the GM antigen in the serum and respiratory tract samples for the diagnostics of CPA has been reported in only a few studies. BAL fluid is theoretically found to be more effective in determining Aspergillus spp. than serum when testing for GM, but the GM antigen is sometimes positive in serum [24]. In a series of patients with aspergilloma, the sensitivity of serum and BAL for GM antigen detection was 38 and 92\%, respectively [9]. In another series of patients with CPA, Kono et al. [10] showed that the detection of GM antigen on bronchial washing specimens was more sensitive $(85.7 \%)$ than in the serum (14.3\%) for a cut-off optical density index value of $\geq 0.5$, though the specificity was very similar (76.3 vs. $78.9 \%$, respectively).

The serum precipitin test detecting antibodies (IgG) against Aspergillus is the most useful diagnostic tool for $\mathrm{CPA}$, and its sole positivity (associated with a suggestive clinical and radiological context) is sufficient to ensure the diagnosis of CPA. Isolation of Aspergillus on direct examination or culture (an infrequent occurrence) is not required for diagnosis when serum Aspergillus precipitins are positive. Consequently, the development of a new anti- $A$. fumigatus IgG enzyme immunoassay (EIA) was a useful diagnostic tool for the detection of Aspergillus spp. antibodies in CPA [25]. Kitasato et al. [26] reported that the A. fumigatus EIA was more sensitive than the Plate$\mathrm{lia}^{\mathrm{TM}}$ Aspergillus EIA for the diagnosis of CPA, even with a new cut-off index of $\geq 0.5$. Otherwise, false-positive reactions are observed with the Platelia Aspergillus EIA in patients with conditions such as pulmonary actinomycosis.

Similar to other diagnostic methods, however, falsenegative results may arise as a result of seronegative patients receiving corticosteroid therapy or due to infection by a species other than A. fumigatus [5].

\section{Exclusion of Other Diagnoses}

Diagnostic criteria for CPA also need to exclude infectious (TB, non-TB mycobacterial infection, histoplasmosis and coccidiomycosis) or non-infectious (lung cancer, rheumatoid arthritis and sarcoidosis) pulmonary disease that can mimic or promote the occurrence of CPA $[3,4]$. Furthermore, recent studies report that patients with CPA may, concurrently or subsequently, also have the above-cited chronic infections [28-30]. Accordingly, before considering the treatment of probable CPA and in 
Fig. 3. The anatomical and clinical continuum of bronchopulmonary Aspergillus spp. infections, according to host immunity. HSP = Hypersensitivity pneumonitis $[2,4]$.

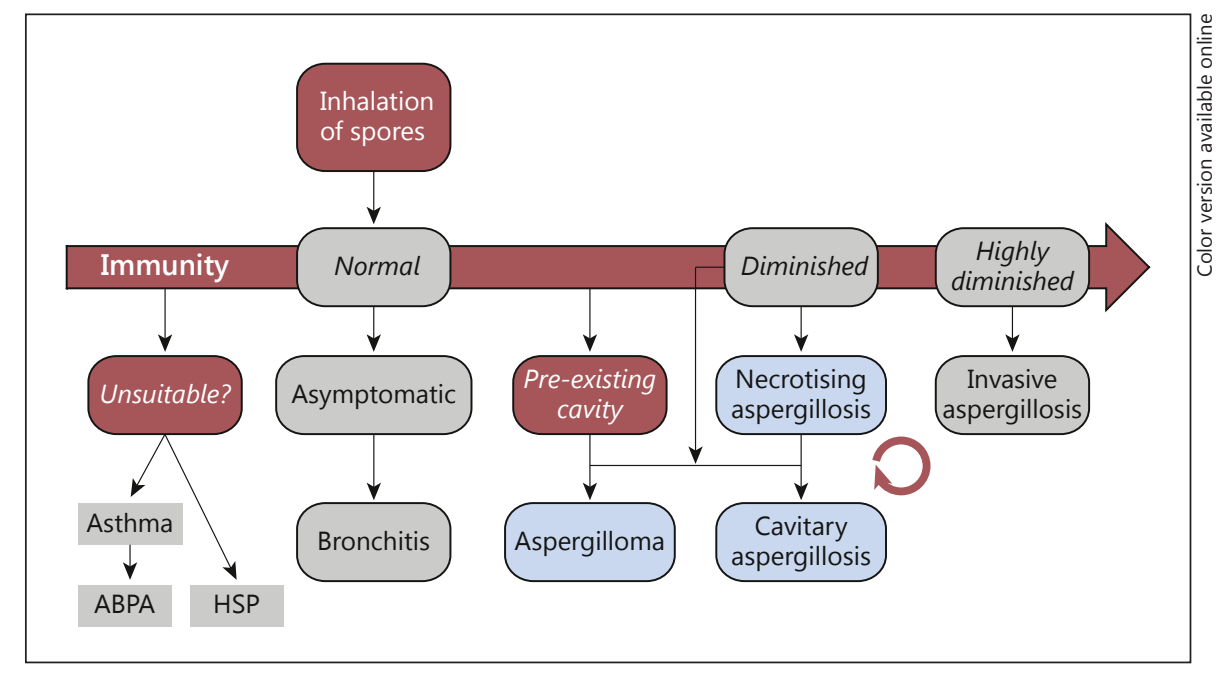

the absence of absolute diagnostic criteria for CPA, it seems necessary to eliminate the diagnosis of lung cancer or other associated infections by any means, e.g. the repetition of microbiological research in the sputum or by the appropriate serologies, but also by performing a fiberoptic bronchoscopy or a new chest CT scan after some weeks of observation.

\section{CPA: Classification}

CPA exists in the form of distinct classifications with regard to clinical, radiological and physiopathological entities. All entities have been found to share the characteristics of weight loss, fatigue and chronic cough, sometimes associated with chest pain and haemoptysis; this suggests that they all belong to a common Aspergillus spp. infectious group [3] (table 1).

Aspergilloma is a form of CPA in which pre-existing cavities become colonised by Aspergillus spp. Aspergilloma has been subdivided into the 'simple' and 'complex' types according to the radiological appearance; the latter is now called 'chronic cavitary pulmonary aspergillosis' (CCPA). According to Denning et al. [3], CPA with systemic symptoms can been subdivided into chronic necrotising pulmonary aspergillosis (CNPA), CCPA and CFPA. As it is clinically difficult to distinguish these forms from one another, we use the term 'CPA' to encompass simple aspergilloma, CCPA and CFPA, with CNPA being classified as a sub-acute form of IPA [31].

Simple aspergilloma is caused by Aspergillus spp., most frequently A.fumigatus, although other species including
A. flavus, A. niger and A. terreus can be responsible for the condition $[7,23,32]$. Colonisation occurs in either scarred lungs or in a pre-existing lung cavity that may have been caused by a previous infection, most commonly TB. Patients are usually asymptomatic, though a varying degree of mild-to-severe haemoptysis is also found [32].

CCPA (formerly called complex aspergilloma) is the formation and expansion of multiple cavities or the extension of a pre-existing cavity; some contain fungal balls $[3,7]$. Pulmonary and systemic symptoms reflect a granulomatous reaction and tissue necrosis, which are destructive for the lung and lead to the formation of cavities through parenchymal necrosis (see the paragraph above: 'The Role of CT Scan Imaging in CPA Diagnosis'). Chronic development of CCPA occurs over several months and the condition commonly relapses, even after surgery. Over time, if untreated, these cavities enlarge and coalesce, and the fungal balls may appear or disappear. The terminal fibrosing evolution of CCPA is CFPA, and this arises when the aspergillosis remains untreated, leaving chronic scars on the lungs and extensive pulmonary fibrosis that does not improve with subsequent treatment $[3,33]$. The development from CCPA to CNPA is caused by cavity expansion and is found among patients with impaired immunity, such as those on low immunosuppressive doses of corticosteroids or those suffering from diabetes or liver cirrhosis (fig. 3) [3].

CNPA is the progressive enlargement of a single (preexisting) cavity, usually with a thin wall, either progressing slowly over months or rapidly in weeks (called the sub-acute form) [3]. This necrotising condition was initially identified as an intermediate entity between a 
Fig. 4. Proposal for a global therapeutic strategy algorithm of CPA. ITCZ = Itraconazole; $\mathrm{L}-\mathrm{AMB}=$ liposomal amphotericin $\mathrm{B}$; POSA = posaconazole; $\mathrm{SA}=$ simple aspergilloma; $\mathrm{VRCZ}$ = voriconazole.

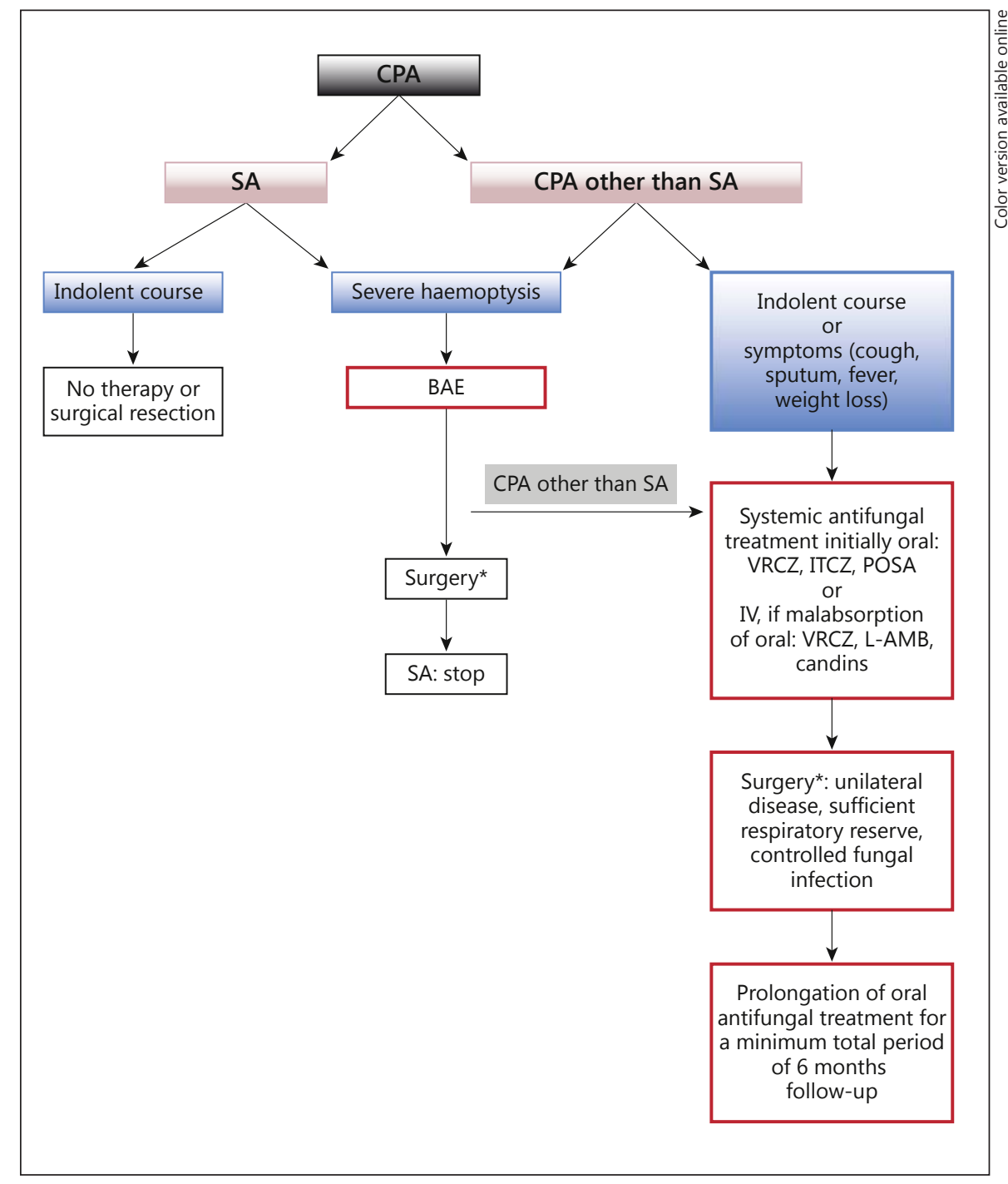

chronic infection and invasive aspergillosis [17]; although these likely share common radiological and clinical features, a distinct type is considered for necrotising subacute IPA, in which the rapidity of installation seems closer to that of invasive forms of aspergillosis in the nonimmunocompromised context $[3,31]$.

A new, simplified classification system encompassing all pulmonary Aspergillus spp. infections has been proposed as the following three entities: (1) simple aspergilloma, (2) CCPA, as symptomatic complex aspergilloma or slowly progressive CNPA ( $>3$ months in duration), occurring in patients with a previous history of bronchopulmonary disease, with pre-existing cavitary and with/ without intracavitary fungal balls and (3) sub-acute IPA (rapidly progressive CNPA of $<3$ months in duration).
However, clinical and radiological descriptions of a large cohort of patients is required to better understand the significance of features of Aspergillus spp. infections in terms of prognosis and outcome [33].

\section{CPA: Current Treatment Options}

The aim of current treatment strategies is to eradicate aspergillosis or, more commonly, to provide palliative treatment. For simple aspergilloma, treatment is not necessarily required, as spontaneous lysis occurs in $10 \%$ of cases and clinical and radiological stabilisation in $25 \%$ [34]. However, surgical resection may be appropriate in selected patients (carefully evaluated for risk factors) [35]. 
Oral systemic antifungal therapy is unnecessary for simple aspergilloma [35]. Evidence from systemic administration of itraconazole found a lack of immunological or symptomatic improvement in patients with pulmonary aspergilloma [36]. The lack of lesion blood supply proves medical therapy to be generally ineffective [37]. However, a late response was observed after itraconazole administration, suggesting that antifungal therapy may indeed have increased efficacy in the long term [36]. Antifungal treatment may be combined with surgery in certain cases of prolonged illness or frequent haemoptysis [38], but this has not been found to offer any overall improvement in patient morbidity or mortality in the last decade [39]. It is also considered hazardous and should only be performed in high-risk patients [40].

For CPA, long-term antifungal treatment is usually required, but case-by-case surgery should be discussed [41]. Patients with poor pulmonary function are generally inoperable and can, alternatively, undergo endovascular treatment such as bronchial artery embolisation (BAE) in the case of life-threatening haemoptysis and also receive systemic antifungal therapy [42]. However, no documented therapeutic strategy has been outlined for CCPA and CNPA because very few methodologically satisfactory studies have been carried out. The general recommendation is prolonged or life long systemic antifungal treatment [35].

In all cases, it is important to treat underlying conditions and comorbidities by reducing corticosteroid therapy and providing nutritional support and respiratory rehabilitation. There are several contributing factors that determine which treatment approach to adopt and these need to be tailored to the individual.

\section{Surgical Therapy for CPA}

According to recent IDSA guidelines, in patients at a low surgical risk, surgical resection can offer a permanent cure as well as avoid haemoptysis and locoregional extension, and therefore improves survival [35, 38, 42]. Possible procedures include lobectomy, pulmonectomy, atypical resection, cavernostomy and thoracoplasty [43]. Surgery has been found to be successful in patients with better lung function and localised pulmonary disease [44]. In those with compromising underlying diseases, impaired respiratory function, poor general health and in the elderly, there is a high frequency of postoperative morbidity and mortality as well as a lack of published randomised clinical trial data $[7,42,43,45]$. Surgical treatment should be considered when the clinical benefit outweighs the risk to the patient [38] and the cost
Table 4. Causes of surgical mortality in 41 patients with pulmonary aspergilloma [46]

\begin{tabular}{lc}
\hline Cause of death & Patients, $\mathrm{n}(\%)$ \\
\hline Respiratory failure & $16(41.0)$ \\
Bleeding & $6(15.4)$ \\
Pneumonia & $3(7.7)$ \\
Underlying disease & $3(7.7)$ \\
Myocardial infarction & $2(5.1)$ \\
Sepsis & $2(5.1)$ \\
Aspergillosis & $2(5.1)$ \\
Miscellaneous & $5(12.8)$ \\
\hline
\end{tabular}

${ }^{1}$ Including bronchopleural fistula [37], empyema, hypoxic encephalopathy, aortic graft thrombosis, disseminated intravascular coagulopathy.

Table 5. Surgical complications in 109 patients with pulmonary aspergilloma [46]

\begin{tabular}{ll}
\hline Types of complications & Patients, $\mathrm{n}(\%)$ \\
\hline Bleeding & $38(22.2)$ \\
Residual pleural space & $33(19.3)$ \\
Bronchopleural fistula & $27(15.8)$ \\
Empyema & $23(13.5)$ \\
Respiratory insufficiency & $14(8.2)$ \\
Air leakage & $11(6.4)$ \\
Wound infection & $5(2.9)$ \\
Sepsis & $3(1.8)$ \\
Pneumonia & $2(1.2)$ \\
Atelectasis & $2(1.2)$ \\
Retained secretions & $2(1.2)$ \\
Miscellaneous & $11(6.4)$ \\
\hline
\end{tabular}

${ }^{1}$ Including pleural effusion, wound dehiscence, lobar infarction, paralytic ileus and unknown complications in 7 patients.

of treatment; this was illustrated in a retrospective study conducted by Chen et al. [46] (tables 4, 5). Farid et al. [47], in a recent study on 30 patients who underwent surgery for CPA (12 with simple aspergillomas and 18 with CCPA), reported that surgery offered good outcomes with acceptable morbidity. No operative or 30day mortality was reported. The main postoperative complications were prolonged air leak (23\%), empyema (20\%) and respiratory failure requiring tracheostomy/ reintubation (13\%). Recurrence of CCPA was noted in 8 patients (29\%), most of whom had had prior CCPA (75\%). 


\section{Systemic Antifungal Treatments for CPA}

Administration of systemic antifungal therapy is, to date, the cornerstone of CPA management, especially in patients who cannot undergo surgery [35]. In a very recent randomised controlled trial, Agarwal et al. [31] reported the superiority of itraconazole therapy over standard supportive treatment. Two other trials have also shown an improvement in quality of life after initiating triazoles in the setting of CPA $[41,55]$. While several different classes of antifungal agents have been introduced, the emergence of triazole antifungal drugs has been found to be an effective therapeutic agent against almost all forms of CPA [48]. If tolerated, triazole agents have acceptable side effects $[8,12,35,36]$. Used orally, these drugs can be particularly suitable for the long-term treatment that is needed for recovery. IV administration is not necessary in the majority of cases, as CPA is usually chronic or sub-acute. It also requires hospitalization and therefore incurs higher costs. In a recent prospective randomized trial, IV administration of micafungin was not significantly different in efficacy to voriconazole [48]. Voriconazole is available in both the oral and IV form, with the oral form being very well absorbed.

\section{Antifungal Treatment, Amphotericin B and Candins}

IV application of amphotericin B was found to achieve success against CPA in $80 \%$ of patients [3] (table 6). However, this response was short-lived and 7/18 of the patients experienced significant toxicity [3]. Other studies have also found amphotericin B to have several associated toxicities and a limited efficacy related to long-term therapy $[53,56]$. Although the amphotericin lipid complex has an improved safety profile, there is no evidence of enhanced efficacy [56].

Micafungin, an echinocandin antifungal agent, has been proposed as an alternative to voriconazole due to its improved safety profile (see below). A multi-centre trial in Japan, conducted by Kohno et al. [51], found the efficacy of the IV administration of micafungin and voriconazole to be 60 and 53.2\%, respectively. However, there was no difference in efficacy between the two after 2 weeks ( $p=0.34)$ or at the end of the treatment $(p=0.5)$. Fewer adverse effects were reported with micafungin [51]. Further study on the safety of micafungin found that adverse effects occurred in $15.8 \%$ of patients, the most common being abnormal liver function. This was a small, non-randomised observational study, however, so further studies are required to establish the safety of this drug in comparison to voriconazole [57]. A weak point of these candins is their exclusive IV formulation availability, which can make administration in the long term more difficult.

\section{Oral Triazoles, Itraconazole, Voriconazole and Posaconazole}

For cases of CCPA and CNPA, oral triazole antifungal therapy is preferred; it improves symptoms and prevents the progressive destruction of lung tissue and the development of pulmonary fibrosis $[50,58]$. Patients with CPA generally require long-term treatment, particularly recommended for CCPA patients due to their local innate immune defects, established during relapses when itraconazole is stopped $[7,42,53]$. This has also been indicated by a higher response rate to long-term treatment (53.3\%) than short-term treatment (25\%) in 23 CNPA patients receiving IV and oral therapy [58].

\section{Itraconazole}

The use of the fungistatical azole itraconazole for CPA has been evaluated, with varying results $[3,36,40,49,60]$. In an open, multi-centre study, 93\% (13/14) of CNPA patients were found to respond to a daily treatment of 200$400 \mathrm{mg}$ of itraconazole; $3 / 13$ had relapses [53] (table 6). In a controlled trial by Agarwal et al. [31], the number of patients showing an overall response was significantly higher in the itraconazole group ( $\mathrm{n}=17 ; 76.5 \%)$ than in the control group $(n=14 ; 35.7 \%)(p=0.02)$. The number of patients demonstrating clinical or radiological response was also significantly higher in the itraconazole group than in the controls $(\mathrm{p}=0.016$ and 0.01 , respectively). While itraconazole has demonstrated higher complete and improved response rates than amphotericin B [53], it has many drug interactions, and recent findings have suggested prolonged treatment may cause resistant A. fumigatus [61]. This was demonstrated by a positive correlation between the duration of treatment and minimum inhibitory concentration (MIC) levels [62]. In addition, itraconazole administration has been associated with cardiac ventricular and gastrointestinal dysfunction and variable bioavailability $[35,49]$.

\section{Voriconazole}

Voriconazole has a different structure to itraconazole and so acquires different properties, including an extended spectrum of activity against rarer fungal species, a different pharmacokinetic profile as well as different drug interactions and adverse effects. It is therefore a useful alternative for patients who do not respond/are intolerant to itraconazole [50,63]. Voriconazole exhibits fungicidal 
Table 6. Responses (clinical improvement and/or complete response) to systemic antifungal treatments: main clinical studies

\begin{tabular}{|c|c|c|c|}
\hline \multirow[t]{2}{*}{ Details of clinical trial/end points } & \multirow{2}{*}{$\begin{array}{l}\text { Patients, } \\
\mathrm{n}\end{array}$} & \multirow[t]{2}{*}{ Antifungal agent } & Patients responding to \\
\hline & & & aspergilloma CCPA CFPA CNPA CPA \\
\hline
\end{tabular}

Open, international study of CPA patients to identify more

18 itraconazole, 100-200 mg daily

accurate subcategories. Many end points were analysed to identify good long-term indicators of medical outcome [3]

Prospective, single-centre, randomised controlled trial, no placebo in $31 \quad$ itraconazole, control arm. Itraconazole vs. supportive treatment. Superiority of $400 \mathrm{mg}$ daily itraconazole therapy vs. standard supportive treatment [31]

Detailed examination of 6 patients with CNPA to update approaches for the diagnosis and treatment of CNPA [40]

Open, international study to access the clinical result of treatment in aspergillosis and aspergilloma patients. Assessed marked improvement and cure between 11 and 780 days [49]
Open, multi-centre French study to access itraconazole as an alternative to amphotericin B in IPA and CNPA patients. Improvement, stability and failure were assessed [53]

Prospective, multi-centre study to examine the efficacy and safety of short- and long-term treatment [58]

Retrospective South Korean study to review treatment for CNPA with different anti-fungal drugs for a median of 6 months. Clinical and radiological improvement were assessed [59]

Retrospective study to assess posaconazole treatment in CPA. Clinical and radiological responses at 6 months were determined [8]

Retrospective, multi-centre study to access voriconazole administered in CPA patients. Clinical, radiological and mycological improvements were assessed every 3 months until the end of treatment [12]

Retrospective study assessing the efficacy and tolerability of treatment in patients intolerant of or failing on itraconazole. Response rate to therapy was $64 \%$ over 3 months [50]

Prospective, open-label, non-comparative, multi-centre study over $2 \quad 71$ years to assess the safety and tolerability of voriconazole in CPA patients. Response was based on a composite of clinical, radiological, serological and mycological criteria [54]

Open, non-comparative multi-centre, prospective study to assess 200 $\mathrm{mg}$ voriconazole administered twice daily in invasive and chronic aspergillosis. Efficacy, safety and tolerability by marked clinical, radiological and mycological improvement were assessed [56]

Prospective, non-comparative trial to assess response rates of voriconazole in CPA patients. The primary end point was global success at 6 months for radiological and mycological improvement [55] 41 voriconazole, $200 \mathrm{mg}$ twice daily

voriconazole,

$43(61)$ patient-specific doses

voriconazole, $200 \mathrm{mg}$ twice daily including itraconazole,

posaconazole, $400 \mathrm{mg}$ twice daily

weight according to

voriconazole,

150-220 mg twice daily voriconazole, per body
$24(77)$

4 (67)

$29(66)$

$13(93)$

$10(44)$

$16(62)$
Randomised, multi-centre, open-label study comparing micafungin with voriconazole. There was no difference in efficacy between therapies after 2 weeks $(p=0.34)$ or at the end of treatment $(p=0.5)$ [51]

97

micafungin, $150-300 \mathrm{mg}$

daily (IV) vs.

voriconazole,

$6 \mathrm{mg} / \mathrm{kg}$ twice on day 1 ,

then $4 \mathrm{mg} / \mathrm{kg}$ twice daily (IV)

Prospective study assessing the clinical efficacy and safety of micafungin-itraconazole therapy. The response rate to the combination therapy was $58.8 \%$ [52]
17 micafungin $150 \mathrm{mg}$ daily and itraconazole $200 \mathrm{mg}$ daily for 1 month

Values are $\mathrm{n}(\%) .{ }^{\mathrm{a}}$ Micafugin; ${ }^{\mathrm{b}}$ voriconazole. 
activity against $A$. fumigatus and has illustrated effectiveness against pulmonary and disseminated aspergillosis in both immunocompromised and immunocompetent patients, and has an MIC concentration comparable to amphotericin B and itraconazole $[12,55,56]$. For these reasons, voriconazole has been found to be an effective agent as a primary or salvage treatment against CPA $[12,56$, 64].

A recent prospective, open, non-comparative multicentre study of CPA patients treated twice daily with 200 $\mathrm{mg}$ of voriconazole, found that the primary end point, measured as global success at 6 months with complete or partial $(\geq 50 \%)$ radiological response and mycological improvement, was achieved in $32 \%$ patients (compared to $80 \%$ in an earlier study) $[55,56]$. This was the first prospective study that included an extensive follow-up period for measuring response rates and quality-of-life improvements. It found that global success after 3 and 6 months did not differ significantly (29 and 32\%, respectively) [55]. It also found that the efficacy of voriconazole was greater in patients with CNPA than in those with CCPA, with success rates at the end of treatment of 58 and $32 \%$, respectively. These findings were supported by Camuset et al. [12] (table 6). The proposed explanation for this may be that the Aspergillus spp. infection in CNPA develops within the parenchymal lung tissue whereas in CCPA, it develops within the cavities surrounded by fibrotic tissue and is therefore less exposed to the antifungal agent [55]. However, in another study, 64\% (7/11) of the CCPA patients showed a response (assessed by a fall in inflammatory markers) to voriconazole treatment after 3 months [50]. The most commonly occurring side effects of voriconazole were visual and gastrointestinal disorders and hepatotoxicity $[12,51,55,56]$.

Hepatotoxicity may be a limiting factor for voriconazole administration and regular monitoring with liver function tests is advised in all patients. Phototoxicity and potentially associated squamous cell carcinoma have also been reported. In the case of phototoxicity, dermatological evaluation should be performed on a regular basis and discontinuation of treatment should be considered [65].

\section{Posaconazole}

The use of itraconazole and voriconazole against CPA has been associated with Aspergillus spp. resistance and drug-related toxicities and has led to the evaluation of alternative drugs [66]. The safety and efficacy of posaconazole $400 \mathrm{mg}$ administered twice daily were assessed in 79 patients with CPA, and a response was observed in $61 \%$ at 6 months and in $46 \%$ at 12 months, comparable with success rates observed for itraconazole and voriconazole administration [8]. In patients where isolates had a posaconazole MIC of $>8 \mathrm{mg}$, there was no response to posaconazole therapy. However, in $50 \%$ of patients who were not anticipated to respond to itraconazole (isolates had an itraconazole MIC of $>8 \mathrm{mg}$ ), a response to posaconazole was still seen. Adverse effects were found in $15 \%$ of patients, with the most commonly occurring effect being nausea [8].

\section{Refinement Is Needed for the Best Therapeutic Choice}

The clinical improvement or complete response to systemic antifungal agents against CPA in several retrospective and prospective studies in the period 1988-2013 are recorded in table 6 . Although the efficacy of systemic antifungal treatments has been assessed, it is difficult to compare the response rates in retrospective studies that involve a small number of patients with highly varied backgrounds [3, 12, 49, 50,53]. These studies also have poorly defined end points and the CPA entities vary between studies $[8,49,53]$. In the voriconazole studies of Jain and Denning [50] and Camuset et al. [12], a stable response was considered as failure; this opposes the results of several other itraconazole studies [36, 53].

The basis of the medical treatment of CPA is long-term treatment with one of the triazole-group drugs, reflecting up-to-date treatment guidelines [35]. However, more prospective studies with greater statistical power are required; these must be comparative and define strategies and treatment durations [35]. According to the current IDSA guidelines, antifungal treatment with itraconazole, voriconazole and posaconazole provides potential for therapeutic benefit with comparatively minimal risk [35].

\section{Endovascular and Other Interventional}

\section{(Instrumental) Procedures}

In the majority of cases, CPA has been associated with haemoptysis. Haemoptysis is the cause of death in up to $26 \%$ of patients with conditions including aspergilloma and complex aspergilloma/CPA [42]. A possible cause of Aspergillus spp.-induced haemoptysis is the mechanical friction between the fungal balls and the blood vessels of the cavity wall [5]. However, the main mechanism is a bronchial artery hypervascularisation surrounding CPA that is responsible for haemoptysis, although the pulmonary artery area can be involved in rare cases of the semiinvasive forms. The severity of haemoptysis is dependent on the underlying disease and, due to the high mortality rate, prompt management and treatment are required [67]. 
BAE acts by blocking the putative vessel that supplies the bleeding site in patients [29]. Evidence has demonstrated that BAE is able to achieve immediate control of haemoptysis in $91.6 \%$ of aspergilloma patients within $24 \mathrm{~h}$ [68]. This has been further supported by Uflacker et al. [69], suggesting that BAE is an effective treatment for CPA patients; however, relapse is common and may be a result of partial embolization, progression of CPA and diffuse pulmonary involvement [69]. Any patient with even minimal haemoptysis should be promptly investigated by CT angiography for a clear understanding of the bleeding site, the underlying disease and the vascular origin of the bleeding.

Localised antifungal therapy has demonstrated efficacy in treating aspergilloma/CPA by using endobronchial or intracavitary instillation of amphotericin B. Trials have shown that $87.5 \%$ of patients achieved resolution or clinical improvement with this treatment [42]. A study conducted by Giron et al. [70] injected amphotericin paste and found that this method controls haemoptysis (with or without previous embolization). The CT-guided injected paste also provided prolonged exposure to the antifungal agent, and led to the disappearance of the aspergilloma and a negative result for Aspergillus spp. serology [71]. This procedure is, however, problematic for those with compromised pulmonary function and can be associated with pulmonary abscess, anaphylactic shock, pneumothorax, haemoptysis and pleural seeding. In addition, although the aspergilloma was found to disappear, the cavity remained in 26 patients and this can cause potential recurrence of aspergilloma in the future $[42,70]$. This procedure should only be used in the treatment of cases refractory to the reference treatment [35].

\section{CPA: Proposal for a Global Therapeutic Strategy Algorithm}

Figure 4 is a tentative global therapeutic strategy for CPA. In all cases, any associated comorbidity, smoking, malnutrition and alcohol intake or diabetes should be considered and monitored as well as the intake of oral and inhaled corticosteroids. All non-mycological causes of infectious or non-infectious pulmonary diseases must be investigated and treated.

The first question to ask is 'Is it a simple aspergilloma or not?' If the answer is yes, and haemoptysis is absent, then surgery or treatment with antifungal agents is not recommended. Regular follow-up with clinical evaluation, chest X-ray and CT scan is needed. For patients with haemoptysis, surgery should be considered in the absence of contraindications. If surgery is undertaken, postopera- tive antifungal treatment is still not recommended for this patient group.

In the case of patients with CPA other than simple aspergilloma, the use of oral triazole treatment may be preferable to IV treatment (good absorption) [31]. For patients with severe haemoptysis, the option of BAE combined with oral triazole treatment should be considered. Subsequently, the patient's tolerance to treatment, improvement in quality of life as well as signs of infection on imaging and Aspergillus in the culture sputum should be assessed, and whether further treatment (3-6 months) is required. The duration of antifungal treatment should be longer in cases of CCPA (at least 6 months) than in CNPA ( $\leq 6$ months). If the initial treatment was found to be inefficient and/or the patient had poor tolerance, the treatment should be stopped and other options considered. Patients in whom problems with oral absorption of the triazoles are suspected should rather receive IV administration, with oral triazoles being reintroduced with dosage monitoring by serum analysis.

In all cases of CPA other than simple aspergilloma, once the patient has undergone pulmonary rehabilitation including renourishment and 1-3 months of systemic treatment with antifungal agents, surgery is the norm. This is to be followed by treatment with antifungal agents for which the indication and duration must be considered depending on: (1) the underlying disease, (2) the persistence of Aspergillus in the pathological analysis and the culture of surgery specimens and (3) the pleural involvement/contamination during surgery. Surgery is preferable for patients where the disease is stable, but not during episodes of active haemoptysis. In patients with haemoptysis, BAE should be considered along with azole treatment and surgery should be performed only after resolution of the haemoptysis.

\section{Summary}

Bronchopulmonary Aspergillus spp. infections include heterogeneous clinical entities which are complicated by associated comorbidities, underlying lung diseases (most commonly severe COPD) and corticosteroid administration. Surgical treatment alone is rarely possible due to the high risk of morbidity and mortality (the optimal candidates for surgical resection are those with a simple aspergilloma and where breathing function is only slightly altered). As a result, a multi-disciplinary approach must be implemented for the treatment of CPA involving specialists including surgeons, radiologists, functional therapists 
and pulmonologists. This will facilitate the establishment of individualised treatment strategies that take into account the complications that are associated with the treatment of the infection and the underlying diseases. In the area of treatment against aspergillosis, antifungal agents are growing fast, especially with the success of efficacy studies on the long-term oral administration of triazoles for the necrotising and/or sub-acute forms of aspergillosis that have been illustrated in the current IDSA guidelines. However, therapeutic sequences in these forms need to be defined by conducting large, adequately powered, prospective trials in order to determine the optimal therapy and duration of treatment.

\section{Acknowledgements}

Editorial support for the preparation of this paper was supplied by HealthCare21 and was funded by Pfizer International Operations.

\section{References}

1 Knutsen AP, Slavin RG: Allergic bronchopulmonary aspergillosis in asthma and cystic fibrosis. Clin Dev Immunol 2011;843-763.

2 Kousha M, Tadi R, Soubani AO: Pulmonary aspergillosis: a clinical review. Eur Respir Rev 2011;20:156-174.

-3 Denning DW, Riniotis K, Dobrashian R, Sambatakou H: Chronic cavitary and fibrosing pulmonary and pleural aspergillosis: case series, proposed nomenclature change and review. Clin Infect Dis 2003;37(suppl 3): S265-S280.

4 Vlahakis NE, Aksamit TR: Diagnosis and treatment of allergic bronchopulmonary aspergillosis. Mayo Clin Proc 2001;76:930-938.

5 Soubani AO, Chandrasekar PH: The clinical spectrum of pulmonary aspergillosis. Chest 2002;121:1988-1999.

6 Smith NL, Denning DW: Underlying conditions in chronic pulmonary aspergillosis including simple aspergilloma. Eur Respir J 2011;37:865-872.

7 Sales MPU: Aspergillosis: from diagnosis to treatment (chapt 5). J Bras Pneumol 2009;35: 1238-1244.

-8 Felton TW, Baxter C, Moore CB, Roberts SA, Hope WW, Denning DW: Efficacy and safety of posaconazole for chronic pulmonary aspergillosis. Clin Infect Dis 2010;51:13831391.

-9 Park SY, Lee SO, Choi SH, Jeong JY, Sung H, Kim MN, Choi CM, Hong SB, Oh YM, Shim TS, Lim CM, Koh Y, Kim DS, Kim YS, Woo JH, Kim SH: Serum and bronchoalveolar lavage fluid galactomannan assays in patients with pulmonary aspergilloma. Clin Infect Dis 2011;52:e149-152.

10 Kono Y, Tsushima K, Yamaguchi K, Kurita N, Soeda S, Fujiwara A, Sugiyama S, Togashi Y, Kasagi S, To M, To Y, Setoguchi Y: The utility of galactomannan antigen in the bronchial washing and serum for diagnosing pulmonary aspergillosis. Respir Med 2013;107: 1094-1100.

11 Izumikawa K, Yamamoto Y, Mihara T, Takazono T, Morinaga Y, Kurihara S, Nakamura S, Imamura Y, Miyazaki T, Nishino T, Tsuka- moto M, Kakeya H, Yanagihara K, Mine M, Yasuoka A, Tashiro T, Kohno S: Bronchoalveolar lavage galactomannan for the diagnosis of chronic pulmonary aspergillosis. Med Mycol 2012;50:811-817.

12 Camuset J, Nunes H, Dombret MC, Bergeron A, Henno P, Philippe B, et al: Treatment of chronic pulmonary aspergillosis by voriconazole in nonimmunocompromised patients. Chest 2007;131:1435-1441.

13 Parakh UK, Sinha R, Bhatnagar AK, Singh P: Chronic necrotising pulmonary aspergillosis: a rare complication in a case of silicosis. In dian J Chest Dis Allied Sci 2005;47:199-203.

14 Pena TA, Soubani AO, Samavati L: Aspergillus lung disease in patients with sarcoidosis: a case series and review of the literature. Lung 2011;189:167-172.

15 Yousem SA: The histological spectrum of chronic necrotizing forms of pulmonary aspergillosis. Hum Pathol 1997;28:650-656.

16 Binder RE, Faling LJ, Pugatch RD, Mahasen C, Snider GL: Chronic necrotizing pulmonary aspergillosis: a discrete clinical entity. Medicine 1982;61:109-124.

17 Gefter WB, Weingrad TR, Epstein DM, Ochs RM, Miller WT: 'Semi-invasive' pulmonary aspergillosis: a new look at the spectrum of Aspergillus infections of the lung. Radiology 1981;140:313-321.

18 Klein DL, Gamsu G: Thoracic manifestations of aspergillosis. Am J Roentgenol 1980;134: 543-552.

19 Kim SY, Lee KS, Han J, Kim J, Kim TS, Choo SW, Kim SJ: Semi-invasive pulmonary aspergillosis: CT and pathologic findings in six patients. Am J Roentgenol 2000;174:795-798.

20 Franquet T, Müller NL, Giménez A, Guembe $\mathrm{P}$, de la Torre J, Bagué S: Spectrum of pulmonary aspergillosis: histologic, clinical, and radiologic findings. Radiographics 2001;21: 825-837.

21 Uffredi ML, Mangiapan G, Cadranel J, Kac G: Significance of Aspergillus fumigatus isolation from respiratory specimens of nongranulocytopenic patients. Eur J Clin Microbiol Infect Dis 2003;22:457-462.
22 Zmeili OS and Soubani AO: Pulmonary aspergillosis: a clinical update. QJM 2007;100: 317-334.

23 Ohba H, Miwa S, Shirai M, Kanai M, Eifuku $\mathrm{T}$, Suda T, et al: Clinical characteristics and prognosis of chronic pulmonary aspergillosis. Respir Med 2012;106:724-729.

24 Izumikawa K, Yamamoto Y, Mihara T, Takazono T, Morinaga Y, Kurihara S, et al: Bronchoalveolar lavage galactomannan for the diagnosis of chronic pulmonary aspergillosis. Med Mycol 2012;50:811-817.

25 Guitard J, Sendid B, Thorez S, Gits M, Hennequin $\mathrm{C}$ : Evaluation of a recombinant antigen-based enzyme immunoassay for the diagnosis of noninvasive aspergillosis. J Clin Microbiol 2012;50:762-765.

26 Kitasato Y, Tao Y, Hoshino T, Tachibana K, Inoshima N, Yoshida M, Takata S, Okabayashi K, Kawasaki M, Iwanaga T, Aizawa $\mathrm{H}$ : Comparison of Aspergillus galactomannan antigen testing with a new cut-off index and Aspergillus precipitating antibody testing for the diagnosis of chronic pulmonary aspergillosis. Respirology 2009;14:701-718.

27 Greene R: The radiological spectrum of pulmonary aspergillosis. Med Mycol 2005; 43:S147-S154.

28 Hafeez I, Muers MF, Murphy SA, Evans EG, Barton RC, McWhinney P: Non-tuberculous mycobacterial lung infection complicated by chronic necrotising pulmonary aspergillosis. Thorax 2000;55:717-719.

29 Kobashi Y, Fukuda M, Yoshida K, Miyashita N, Niki Y, Oka M: Chronic necrotizing pulmonary aspergillosis as a complication of pulmonary Mycobacterium avium complex disease. Respirology 2006;11:809-813.

30 Jhun BW, Jeon K, Eom JS, Lee JH, Suh GY, Kwon OJ, Koh WJ: Clinical characteristics and treatment outcomes of chronic pulmonary aspergillosis. Med Mycol 2013;51:811-817.

- 31 Agarwal R, Vishwanath G, Agarwal AN, Garg M, Gupta D, Chakrabarti A: Itraconazole in chronic cavitary pulmonary aspergillosis: a randomised controlled trial and systematic review of literature. Mycoses 2013;56:559-570. 
-32 Al-Alawi A, Ryan CF, Flint JD, Müller NL: Aspergillus-related lung disease. Can Respir J 2005; 12:377-387.

33 Philippe B, Germaud P: Aspergillus in the immunocompetent patient. Towards a new classification? Rev Mal Respir 2005;22:711-714.

-34 Denning DW: Chronic forms of pulmonary aspergillosis. Clin Microbiol Infect 2001;7: 25-31.

-35 Walsh TJ, Anaissie EJ, Denning DW, Herbrecht R, Kontoyiannis DP, Marr KA, et al: Treatment of aspergillosis: clinical practice guidelines of the Infectious Diseases Society of America. Clin Infect Dis 2008;46:327-360.

- 36 Campbell JH, Winter JH, Richardson MD, Shankland GS, Banham SW: Treatment of pulmonary aspergilloma with itraconazole. Thorax 1991;46:839-841.

- 37 Passera E, Rizzi A, Robustellini M, Rossi G, Della Pona C, Massera F, et al: Pulmonary aspergilloma: clinical aspects and surgical treatment outcome. Thorac Surg Clin 2012;22 345-361.

- 38 Lee KH, Ryu SM, Park SM, Park HS, Lee KH, Cho SJ: A surgically treated case of chronic necrotizing aspergillosis with pleural invasion. Korean J Thorac Cardiovasc Surg 2012; 45:56-59.

-39 Pabst S, Krüger M, Skowasch D, Zhou H, Bürmann J, Kaminski M: Pulmonary aspergillosis: therapeutic management and prognostic factors from 16 years of monocenter experience. Adv Exp Med Biol 2013;755:225-236.

-40 Saraceno JL, Phelps DT, Ferro TJ, Futerfas R, Schwartz DB: Chronic necrotizing pulmonary aspergillosis - approach to management. Chest 1997;112:541-548.

41 Al-Shair K et al: Long-term antifungal treatment improves health status in patients with chronic pulmonary aspergillosis: a longitudinal analysis. Clin Infect Dis 2013;57:828-835.

-42 Stevens DA, Kan VL, Judson MA, Morrison VA, Dummer S, Denning DW, et al: Practice guidelines for diseases caused by Aspergillus. Clin Infect Dis 2000;30:696-709.

-43 Endo S, Sohara Y, Murayama F, Yamaguchi T, Hasegawa T, Tezuka K, et al: Surgical outcome of pulmonary resection in chronic necrotizing pulmonary aspergillosis. Ann Thorac Surg 2001;72:889-894.

-44 Babatasi G, Massetti M, Chapelier A, Fadel E, Macchiarini P, Khayat A, et al: Surgical treatment of pulmonary aspergilloma: current outcome. J Thorac Cardiovasc Surg 2000;19: 906-912.

-45 Ikemoto H: Medical treatment of pulmonary aspergilloma. Intern Med 2000;39:191-192.

46 Chen JC, Change YL, Luh SP, Lee JM, Lee YC: Surgical treatment for pulmonary aspergilloma: a 28-year experience. Thorax 1997;52: 810-813.
47 Farid S, Mohamed S, Devbhandari M, Kneale M, Richardson M, Soon SY, Jones MT, Krysiak P, Shah R, Denning DW, Rammohan $\mathrm{K}$ : Results of surgery for chronic pulmonary aspergillosis, optimal antifungal therapy and proposed high risk factors for recurrence - a National Centre's experience. J Cardiothorac Surg 2013;8:180.

48 Bulpa P, Dive A, Sibille Y: Invasive pulmonary aspergillosis in patients with chronic obstructive pulmonary disease. Eur Respir 2007;30:782-800.

49 De Beule K, De Doncker P, Cauwenbergh G, Koster M, Legendre R, Blatchford N, et al: The treatment of aspergillosis and aspergilloma with itraconazole, clinical results of an open international study (1982-1987). Mycoses 1988;31:476-485

50 Jain LR, Denning DW: The efficacy and tolerability of voriconazole in the treatment of chronic cavitary pulmonary aspergillosis. Infection 2006;52:133-137.

-51 Kohno S, Izumikawa K, Ogawa K, Kurashima A, Okimoto N, Amitani R, et al: Japan Chronic Pulmonary Aspergillosis Study Group (JCPASG): Intravenous micafungin versus voriconazole for chronic pulmonary aspergillosis: a multicentre trial in Japan. J Infect 2010;61:410-418.

52 Fujita M, Tao Y, Kajiki A, Ouchi H, Harada E, Ikegame $S$, et al: The clinical efficacy and safety of micafungin-itraconazole combination therapy in patients with pulmonary aspergilloma. J Infect Chemother 2012;18:668-674.

53 Dupont B: Itraconazole therapy in aspergillosis: study in 49 patients. J Am Acad Dermatol 1990;23:607-614.

54 Saito T, Fujiuchi S, Tao Y, Sasaki Y, Ogawa K, Suzuki K, et al: Efficacy and safety of voriconazole in the treatment of chronic pulmonary aspergillosis: experience in Japan. Infection 2012;40:661-667.

55 Cadranel J, Philippe B, Hennequin C, Bergeron A, Bergot E, Bourdin A, et al: Voriconazole for chronic pulmonary aspergillosis: a prospective multicentre trial. Eur J Clin Microbiol Infect Dis 2012;31:3232-3239.

56 Sambatakou H, Dupont B, Lode H, Denning DW: Voriconazole treatment for subacute invasive and chronic pulmonary aspergillosis. Am J Med 2006;119:527.e17-e24.

57 Kohno S, Izumikawa K, Kakeya H, Miyazaki Y, Ogawa K, Amitani R, et al: Clinical efficacy and safety of micafungin in Japanese patients with chronic pulmonary aspergillosis: a prospective observational study. Med Mycol 2011;49:688-693.

58 Yoshida K, Kurashima A, Kamei K, Oritsu M, Ando T, Yamamoto T, et al: Efficacy and safety of short- and long-term treatment of itraconazole on chronic necrotizing pulmonary aspergillosis in a multicenter study. J Infect Chemother 2012;18:378-385.
59 Nam HS, Jeon K, Um SW, Suh GY, Chung $\mathrm{MP}, \mathrm{Kim} \mathrm{H}$, et al: Clinical characteristics and treatment outcomes of chronic necrotizing pulmonary aspergillosis: a review of 43 cases. Int J Infect Dis 2010;14:e479-e482.

60 Tsubura E: Multicenter clinical trial of itraconazole in the treatment of pulmonary aspergilloma. Pulmonary Aspergilloma Study Group (in Japanese). Kekkaku 1997;72:557564.

61 Escribano P, Recio S, Peláez T, GonzálezRivera M, Bouza E, Guinea J: In vitro acquisition of secondary azole resistance in Aspergillus fumigatus isolates after prolonged exposure to itraconazole: presence of heteroresistant populations. Antimicrob Agents Chemother 2012;56:174-178.

- 62 Tashiro M, Izumikawa K, Hirano K, Ide S, Mihara T, Hosogaya N, et al: Correlation between triazole treatment history and susceptivity in clinically isolated Aspergillus fumigatus. Antimicrob Agents Chemother 2012;56: 4870-4875.

63 Cecil JA, Wenzel RP: Voriconazole: a broadspectrum triazole for the treatment of invasive fungal infections. Expert Rev Hematol 2009;2:237-254.

64 Limper AH, et al: An official American Thoracic Society statement: treatment of fungal infections in adult pulmonary and critical care patients. Am J Respir Crit Care Med 2011;183:96-128.

65 Pfizer: Summary of Product Characteristics. Vfend (voriconazole).

66 Izumikawa K, et al: Management of chronic pulmonary aspergillosis. Ann NY Acad Sci 2012;1272:40-48.

67 Khalil A, Parrot A, Nedelcu C, Fartoukh M, Marsault C, Carette MF: Severe hemoptysis of pulmonary arterial origin: signs and role of multidetector row CT angiography. Chest 2008; 133:212-219.

68 Corr P: Management of severe hemoptysis from pulmonary aspergilloma using endovascular embolization. Cardiovasc Intervent Radiol 2006;29:807-810.

69 Uflacker R, Kaemmerer A, Picon PD, Rizzon CF, Neves CM, Oliveira ES, et al: Bronchial artery embolization in the management of hemoptysis: technical aspects and long-term results. Radiology 1985;157:637-644.

70 Giron J, Poey C, Fajadet P, Sans N, Fourcade D, Senac JP, et al: CT-guided percutaneous treatment of inoperable pulmonary aspergilloma: a study of 40 cases. Eur J Radiol 1998; 28:235-242.

-71 Giron JM, Poey CG, Fajadet PP, Balagner GB, Assoun JA, Richardi GR, et al: Inoperable pulmonary aspergilloma: percutaneous CTguided injection with glycerin and amphotericin B paste in 15 cases. Radiology 1993; 188: 825-827. 